\title{
DESENVOLVIMENTO DE COMPLEMENTO QGIS PARA PROCESSAMENTO DE MAPAS MORFOMÉTRICOS
}

\author{
Fabrício Rosa Amorim \\ Universidade Federal do Paraná - UFPR, Curso de Pós-Graduação em Ciências Geodésicas, Curitiba, PR, Brasil \\ fabricioamorimeac@hotmail.com
}

Universidade Federal do Paraná - UFPR, Curso de Pós-Graduação em Ciências Geodésicas, Curitiba, PR, Brasil
marcio.schmidt@gmail.com

Niédja Sodré de Araújo

Universidade Federal do Paraná - UFPR, Curso de Pós-Graduação em Ciências Geodésicas, Curitiba, PR, Brasil niedja.geo@gmail.com

Luciene Stamato Delazari

Universidade Federal do Paraná - UFPR, Curso de Pós-Graduação em Ciências Geodésicas, Curitiba, PR, Brasil luciene@ufpr.br

\begin{abstract}
RESUMO
Este trabalho tem por objetivo descrever os procedimentos para aquisição de dados de elevação, bem como descrever o processamento desses dados para a produção de mapas morfométricos para o território brasileiro. Os dados de elevação trabalhados nessa pesquisa são um Modelo Digital de Elevação (MDE) em formato raster e o MDE Topodata, oriundo do Banco de Dados Morfométricos do Brasil. Para tanto, utilizou-se o software QGIS para hospedar o sistema de processamento desenvolvido, assim, abarcando a interface para manipulação dos dados pelos usuários. O processo para a produção dos mapas se deu a partir de implementação de códigos de processamento em linguagem python no software Aton. Os resultados apontam a extensa gama de produtos que podem ser gerados com técnicas de implementação de códigos de geoprocessamento por meio dos softwares utilizados. Esse trabalho irá subsidiar o planejamento e a gestão territorial por meio da análise de variáveis do relevo, bem como facilitará e servirá de base para futuros estudos nessa temática.
\end{abstract}

Palavras-chave: MDE. Topodata. QGIS. Geoprocessamento. Gestão Territorial.

\section{DEVELOPMENT OF QGIS COMPLEMENT FOR PROCESSING MORPHOMETRIC MAPS}

\begin{abstract}
This research aims to describe the procedures for acquiring elevation data, as well as describing their processing to produce morphometric maps for the Brazilian territory. The elevation data worked on in this research are a Digital Elevation Model (DEM) in raster format and a DEM Topodata, from the Brazilian Morphometric Database. For that, the QGIS software has been used to host the processing system developed, thus encompassing the interface for data manipulation by users. The process for producing the maps took place from the implementation of processing python codes in the Aton software. The results point to the extensive range of products that can be generated with techniques for implementing geoprocessing codes in the software used. This paper may subsidize territorial planning and management through the analysis of relief variables, as well as facilitating and serving as a basis for future studies on this topic.
\end{abstract}

Keywords: DEM. Topodata. QGIS. Geoprocessing. Territorial Management. 


\section{INTRODUÇÃO}

O relevo é o elemento fundamental da paisagem física (GUERRA e GUERRA, 2003). A compreensão do meio físico pode requerer a medição e o mapeamento de variáveis morfométricas, tais como: altitude, declividade, curvaturas vertical e horizontal, orientação de vertentes. O estudo dos dados altimétricos baseado em variáveis morfométricas pode ser empregado nas análises ambientais, tal como ocorre na caracterização de unidades da paisagem (HENGL e REUTER, 2009; LANG e BLASCHKE, 2009). Há um grande número de habilidades e resultados de aprendizagem relacionados à representação do relevo em mapas: conhecimento dos elementos do mapa e das formas de representação do relevo; modelagem do relevo para representá-lo como um ambiente natural; conhecimento e manejo das ferramentas computacionais para o cálculo; representação cartográfica de elementos geomorfológicos; reconstrução tridimensional e modelagem virtual; conhecimento dos processos e formas de relevo da terra para sua integração na análise da paisagem e planejamento (IMHOF, 2015).

Por um longo período, as metodologias de análise do relevo foram desenvolvidas com o subsídio da interpretação visual (DENT e YOUNG, 1981). No entanto, existia o desafio de interpretar informações altimétricas, representadas de forma bidimensional através de diferentes técnicas cartográficas (GOBERT, 2005). A partir do avanço tecnológico, as metodologias de análise e representação do relevo vêm sendo realizadas em ambiente computacional de forma a amenizar a demanda de trabalho manual e a subjetividade dessas tarefas (GILES e FRANKLIN, 1998). A computação digital automatizada fornece as medidas de formas da superfície, de contexto, de padrões e de textura que podem ser utilizadas como substitutas aos critérios considerados na interpretação visual de imagens ou fotografias aéreas (HENGL e REUTER, 2009). Para tanto, o trabalho de derivação de dados topográficos pode ser executado com relativa operacionalidade e alta demanda de dados, visto que antes era exigente em tempo e requeria habilidade de pessoas em interpretar fotos aéreas (fotoleitura).

Os Sistemas de Informação Geográfica (SIG) auxiliam nas funções de armazenar, manusear, acessar e processar a informação base da cartografia em ambiente computacional (DE SMITH; GOODCHILD; LONGLEY, 2007). A disponibilidade crescente de bases topográficas digitais, aliada ao uso de SIG, tem impulsionado o desenvolvimento de métodos automáticos de extração de variáveis morfométricas e podem dar suporte à classificação multivariada da paisagem (MOORE; LEWIS, GALLANT, 1993; GILES e FRANKLIN, 1998; MIZUKOSHI e ANIYA, 2002). Entre as variáveis morfométricas que se encontram providas de algoritmos para sua extração, destacam-se: as curvas de nível; a declividade; a orientação de vertentes; a curvatura horizontal; a curvatura vertical; e a identificação de canais de drenagem e divisores de água (VALENZUELA, 1991; GILES e FRANKLIN, 1998; MCMASTER, 2002; VALERIANO, 2002a; VALERIANO, 2002b; VALERIANO, 2003; VALERIANO e CARVALHO JÚNIOR, 2003). Contudo, geomorfólogos e cartógrafos podem utilizar os produtos derivados das variáveis morfométricas para quantificar e representar a forma ou a estrutura de superfícies topográficas por meio de processos semiautomáticos (ROBINSON et al., 1995; WILSON e GALLANT, 2000).

Para fins de mapeamento geomorfológico, em ambiente computacional necessita-se que haja a transformação de informações contínuas (presentes na superfície da Terra) em unidades regionalizadas de relevo, geralmente representadas em pixels (DRĂGUT e EISANK, 2011). Os Modelos Digitais de Elevação (MDE) se referem aos valores altimétricos correspondentes aos objetos existentes na superfície (EL-SHEIMY; VALEO; HABIB, 2005). Os MDE são muito utilizados por proverem coberturas contínuas para áreas extensas e com relativo baixo custo. Existem diversas formas de construção de MDE, dentre as quais: modelos vetorizados ou digitalizados de curvas de nível e/ou pontos cotados do terreno a partir de cartas topográficas; técnicas de levantamento de campo; técnicas de Fotogrametria; interferometria por radar e Sensoriamento Remoto. No Brasil, o Instituto Nacional de Pesquisas Espaciais (INPE) propôs um outro MDE obtido pela reamostragem do MDE SRTM (Shuttle Radar Topography Mission) para criar uma grade de 1" (ou cerca de $30 \mathrm{~m}$ ) com uma abordagem de interpolação geoestatística por krigagem (VALERIANO, 2005; VALERIANO, 2008; VALERIANO; ROSSETTI; ALBUQUERQUE, 2009; VALERIANO e ROSSETTI, 2011). Os dados topográficos obtidos pela reamostragem do MDE SRTM compuseram um banco de dados geomorfométricos do Brasil, denominado Topodata (BRASIL, 2008).

A Cartografia tem experimentado o efeito das novas tecnologias por meio de softwares, os quais possibilitam representar mapas de relevo de forma prática, interativa e detalhada. Torna-se necessário analisar, além dos aspectos técnicos, os aspectos mais sutis da representação que podem enriquecer 
a compreensão dos usuários. Este trabalho objetiva descrever o projeto, a produção e a validação de uma ferramenta (plugin) geoespacial para aquisição e processamento de dados de altitude em ambiente SIG. Para tanto, utilizou-se o MDE Topodata como fonte dos dados altimétricos, ainda que o plugin desenvolvido possibilite a utilização de outros MDE em formato raster. As seguintes representações cartográficas de relevo foram projetadas: curvas de nível; declividade; aspecto (direção das vertentes); relevo sombreado (sombreamento); índice de posição topográfica; índice de rugosidade do terreno; bacias hidrográficas; canais; junções dos canais. Busca-se, a caráter experimental, evidenciar a relevância dos diferentes produtos cartográficos que podem ser gerados a partir de MDE em ambientes de SIG.

\section{MATERIAIS}

Vislumbrou-se neste trabalho o desenvolvimento de um complemento (plugin) para o QGIS para aquisição de MDE Topodata e processamento de mapas a partir de variáveis morfométricas. Para tal, utilizou-se uma base de dados e três softwares livres com diferentes ferramentas, ícones e bibliotecas de linguagem de programação, mais especificamente:

- Base de Dados: banco de dados geomorfométricos do Brasil (Topodata, 2020); malha municipal do Brasil (IBGE, 2020)

- Softwares: QGIS (complementos: Processing Modeler; Plugin Reloader; Plugin Builder); Atom (bibliotecas python: PyQt4.QtCore; PyQt4.QtGui; Osgeo; Stringio; Zipfile; Requests; Gdal; Processing; Psycopg2; pyUFbr); Qt Designer (ícones: Labels; QDialogBox; QLineEdit; SpinBox; QFileDialog).

\section{Desenvolvimento de sistemas de processamento no QGIS}

O QGIS possui um modelador de rotinas de processamento denominado modelador gráfico, o qual permite ao usuário definir os fluxos de trabalho a serem executados como uma etapa única. Basicamente, torna-se necessário definir os dados de entrada, os parâmetros da ferramenta de processamento e os dados de saída para cada produto a ser gerado. Para os dados de entrada, é possível definir os formatos de arquivos: raster, vetor; string; tabela; número; booleano. O modelador gráfico do QGIS inclui algoritmos com parâmetros de processamento, tais como: GDAL, GRASS, SAGA e LAS Tools (processamento Lidar). Os formatos dos arquivos de saída estarão em conformidade com os parâmetros de processamento pré-estabelecidos. O resultado da rotina de processamento construída no modelo de processamento é uma interface disponível na tela principal do QGIS. Dessa maneira, torna-se possível realizar as tarefas de processamento de dados para produção de mapas como uma tarefa única, bem como compartilhar a rotina desenvolvida com outros usuários para que um mesmo padrão de processamento possa ser aplicado em diversos dados. Além da interface de processamento produzida pelo modelador gráfico, é possível exportar o resultado como um script (linhas de processamento) em linguagem de programação python. Para tanto, as operações de processamento podem ser desenvolvidas com novos parâmetros, além de possibilitar a criação de um novo ambiente para hospedar o sistema de processamento.

O complemento do QGIS denominado Plugin Builder pode ser utilizado para o desenvolvimento de complementos (plugins). Para tanto, esse complemento inicialmente visualizado como um formulário cadastra as informações técnicas do produto a ser elaborado, bem como é responsável pela criação dos arquivos necessários para o funcionamento do mesmo. Posteriormente à criação do complemento, faz-se necessário recarregar o plugin no QGIS com o auxílio do complemento Plugin Reloader sempre que forem realizadas alterações na estrutura do complemento. Dessa maneira, é possível alterar o código de um complemento e tê-lo refletido no QGIS.

Para o desenvolvimento do complemento QGIS, utilizou-se o complemento Plugin Builder para gerar os arquivos necessários para a construção do complemento pretendido neste trabalho. Posteriormente, o modelador gráfico foi utilizado para definição das operações a serem executadas por meio das ferramentas disponíveis no QGIS. Em decorrência da definição das ferramentas de processamento especificadas no modelador gráfico e após testar as operações com o auxílio da interface produzida no QGIS, exportou-se a rotina como um arquivo python para posterior implementação das linhas de comando. Para tanto, a etapa da implementação das linhas de código do complemento ocorreu com o 
auxílio do software Atom e de algumas bibliotecas python. Uma biblioteca python é uma coleção de módulos python organizados como um pacote. No geral, isso significa que todos os módulos vivem dentro do mesmo diretório e que esse diretório está armazenado em um dos caminhos de busca que o python realiza para carregá-lo. A Biblioteca Padrão do python é uma coleção de módulos de script acessíveis a um programa python para simplificar o processo de programação e remover a necessidade de reescrever os comandos mais usados. Essas bibliotecas podem ser usadas importando-as no início de um script. Destacam-se as seguintes bibliotecas padrão do python: sys, os, urllib e re. Além desse tipo de biblioteca, é possível encontrar bibliotecas de python voltadas para a ciência de dados (data Science), ou seja, para manipulação, processamento e limpeza de dados para análises complexas.

\section{Desenvolvimento de complemento QGIS}

Nessa etapa, definiu-se a obrigatoriedade do usuário definir a extensão do local onde serão realizadas as operações de processamento a partir de um MDE. Para tal, três opções são disponíveis, sendo: a primeira, a partir de uma camada vetorial (tipo polígono) previamente aberta na tela do QGIS; a segunda, a partir de um arquivo vetorial (tipo polígono) armazenado no computador, onde será realizada uma busca ao arquivo; e a terceira, a partir da seleção de uma unidade da federação e um município pertencente à mesma. No caso da terceira opção ser selecionada, o sistema utiliza a biblioteca python pyUFbr para listar os estados e municípios do Brasil, enquanto a delimitação do município (arquivo vetorial do tipo polígono) é proveniente da malha municipal fornecida pelo IBGE (IBGE, 2020).

Os dados altimétricos oriundos de um MDE podem ser utilizados de duas maneiras, sendo: a primeira opção permite ao usuário inserir manualmente o seu próprio MDE, desde que o mesmo esteja em formato matricial; e a segunda opção permite ao usuário adquirir um MDE diretamente do sítio Topodata. A obtenção dos dados de elevação pela segunda opção ocorre pelo sítio do banco de dados geomorfométricos do Brasil, Topodata (http://www.dsr.inpe.br/topodata/data/geotiff/). Os formatos de arquivos ASCII, GRD, GeoTiff e BMP podem ser encontrados no sítio desse projeto. Esse banco de dados está estruturado em quadrículas compatíveis com a articulação 1:250.000 e estão identificados em folhas pelo prefixo de seis letras "LAHLON", sendo: LA a latitude do canto superior esquerdo da quadrícula; H o hemisfério (S para Sul, ou N para Norte); e LON a longitude (sufixo nn_para nn graus inteiros, ou sufixo nn5 para nn graus e $30^{\prime}$ ). As diferentes variáveis morfométricas disponíveis no sítio estão identificadas por sufixos de duas letras que complementam o prefixo da olha. Para as imagens (GeoTiff) com os dados de altitude (utilizadas nesse trabalho), o arquivo possui a identificação ZN ao final do nome do arquivo. A implementação da opção de aquisição direta do MDE Topodata ocorreu por meio da utilização de bibliotecas python, as quais são descritas no Quadro 1.

\section{Quadro 1 - Bibliotecas Python utilizadas para aquisição do MDE Topodata}

\begin{tabular}{|l|l|}
\hline \multicolumn{1}{|c|}{ Biblioteca } & \multicolumn{1}{c|}{ Descrição } \\
\hline StringlO & Lê e escreve strings (textos) como arquivos \\
\hline Zipfile & Manipulação de arquivos compactados \\
\hline Requests & Aquisição de arquivos a partir de endereços url \\
\hline \multicolumn{2}{|c}{ Fonte - Elaborado pelos autores. }
\end{tabular}

Após a aquisição dos dados de entrada, os mesmos podem ser processados conforme linhas de código para gerar os arquivos finais. Para realizar o processamento dos MDE, a camada de extensão deve estar no mesmo sistema de projeção de coordenadas do MDE, logo, definiu-se as operações para ocorrer essa compatibilização. Após o ajuste dos sistemas de projeções dos arquivos de entrada, definiu-se a operação para recortar o MDE por meio da camada limite. O resultado dessa operação é um MDE reprojetado e recortado conforme a área pretendida pelo usuário. Posteriormente, as operações de processamento para gerar os produtos a partir de um MDE foram definidas (Figura 1). 
Figura 1 - Arquitetura do sistema.

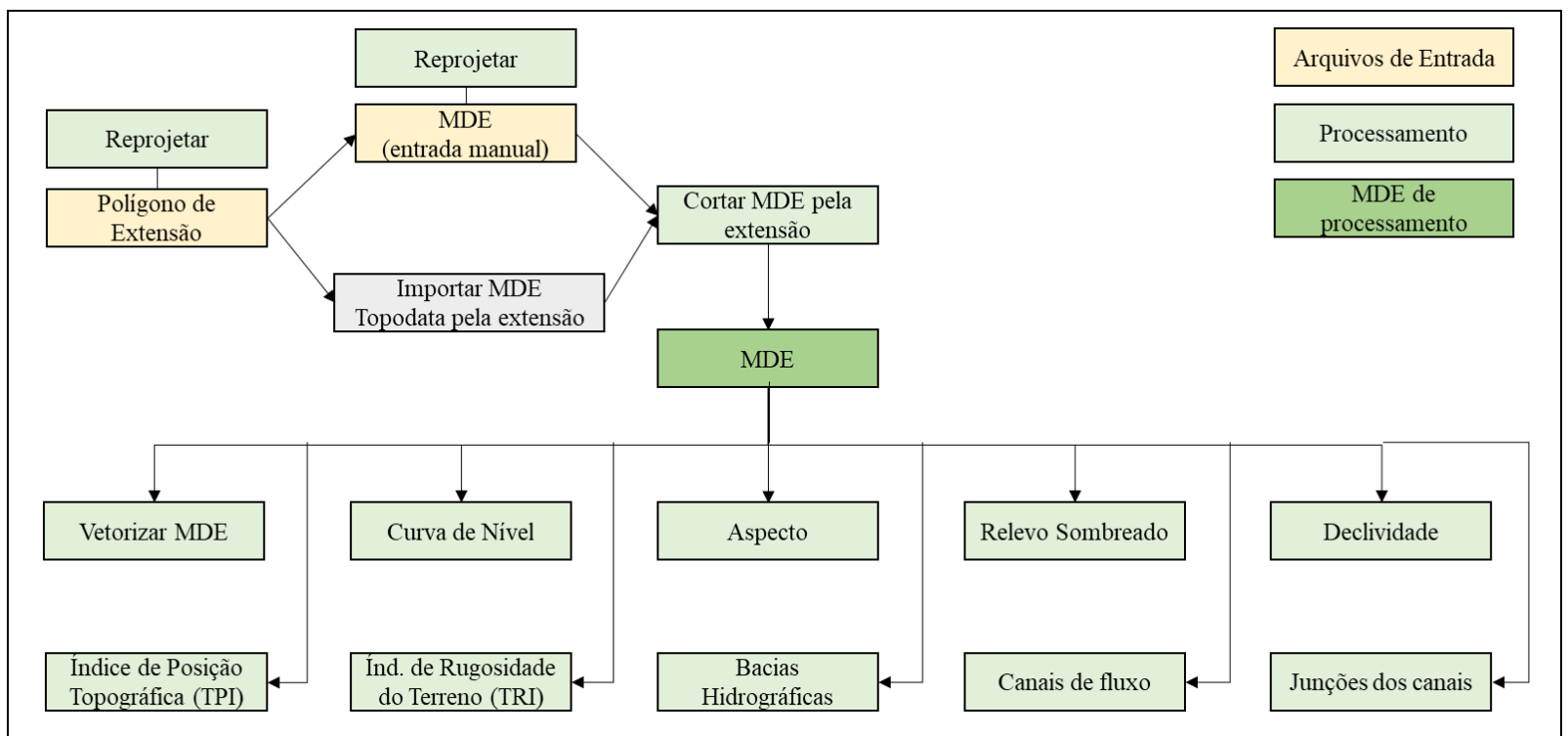

Fonte - Elaborado pelos autores.

Os arquivos finais, em formato matricial ou vetorial, são especificados como: MDE vetorizado; Curvas de nível; Aspecto; Relevo sombreado (sombreamento); Declividade; Índice de Posição Topográfica (Topographic Position Index - TPI); Índice de Rugosidade do Terreno (Terrain Ruggedness Index - TRI); Localização das bacias hidrográficas; Localização dos canais de fluxo hídrico; Localização das junções dos canais de fluxo hídrico. Para tanto, esses arquivos carecem de parâmetros de processamento para definir suas características, assim, os mesmos precisam ser pré-definidos. As bibliotecas python utilizadas nessa etapa são descritas no Quadro 2, bem como os parâmetros de processamento utilizados.

Para o desenvolvimento final da interface do sistema, utilizou-se o software Qt Designer, o qual está disponível em conjunto com o software QGIS e objetiva projetar e criar interfaces gráficas de usuário (Graphical User Interface - GUI) com Qt Widgets. No Qt Designer, torna-se possível compor, personalizar e testar interfaces. Os widgets e formulários criados com o Qt Designer se integram ao código programado. Dessa maneira, as propriedades definidas no Qt Designer podem ser alteradas dinamicamente dentro do código desenvolvido previamente. 
Quadro 2 - Bibliotecas python e parâmetros de processamento

\begin{tabular}{|c|c|c|}
\hline Tarefas & Bibliotecas & Parâmetros \\
\hline Reprojetar & $\begin{array}{l}\text { GDAL (gdalogr: } \\
\text { gdalwarp; } \\
\text { gdalreproject) }\end{array}$ & $\begin{array}{l}\text { Arquivo de entrada (vetor e raster); sistema de referência de } \\
\text { entrada; sistema de referência de saída; nome do arquivo de } \\
\text { saída (raster / vetor) }\end{array}$ \\
\hline Recortar & $\begin{array}{l}\text { GDAL (gdalogr: } \\
\text { gdalintersect) }\end{array}$ & $\begin{array}{l}\text { Arquivo de entrada; arquivo de delimitação; nome do arquivo } \\
\text { de saída (raster) }\end{array}$ \\
\hline Vetorizar MDE & $\begin{array}{l}\text { GDAL (gdalogr: } \\
\text { gdal2xyz) }\end{array}$ & nome do arquivo de saída (vetor). \\
\hline Curvas de nível & $\begin{array}{l}\text { GDAL } \\
\text { (gdalogr:contour) }\end{array}$ & $\begin{array}{l}\text { nome do arquivo de saída (vetor); nome da coluna contendo } \\
\text { os dados de altitude no arquivo resultante; equidistância } \\
\text { pretendida entre as curvas de nível. }\end{array}$ \\
\hline Declividade & $\begin{array}{l}\text { GDAL (gdalogr: } \\
\text { gdal:slope; slope) }\end{array}$ & $\begin{array}{l}\text { nome do arquivo de saída (raster); declividade em } \\
\text { porcentagem; utilizar a fórmula de ZevenbergenThorne para } \\
\text { processamento; computar as bordas da imagem. }\end{array}$ \\
\hline $\begin{array}{l}\text { Mapa } \\
\text { aspecto }\end{array}$ & $\begin{array}{l}\text { GDAL (gdalogr: } \\
\text { aspect) }\end{array}$ & $\begin{array}{l}\text { nome do arquivo de saída (raster); computar as bordas da } \\
\text { imagem; utilizar a fórmula de ZevenbergenThorne para } \\
\text { processamento; representar o mapa pelo ângulo } \\
\text { trigonométrico (ao invés de azimute). }\end{array}$ \\
\hline $\begin{array}{l}\text { Relevo } \\
\text { sombreado }\end{array}$ & $\begin{array}{l}\text { GDAL (gdalogr: } \\
\text { hillshade) }\end{array}$ & $\begin{array}{l}\text { nome do arquivo de saída (raster); computar as bordas da } \\
\text { imagem; utilizar a fórmula de ZevenbergenThorne para } \\
\text { processamento; exagero vertical; escala (razão entre as } \\
\text { unidades horizontal e vertical); ângulo do azimute de } \\
\text { iluminação; altura de iluminação. }\end{array}$ \\
\hline $\begin{array}{l}\text { Índice de } \\
\text { Posição } \\
\text { Topográfica } \\
\text { (TPI) }\end{array}$ & $\begin{array}{l}\text { GDAL (gdalogr: } \\
\text { tpi) }\end{array}$ & $\begin{array}{l}\text { nome do arquivo de saída (raster); computar as bordas da } \\
\text { imagem. }\end{array}$ \\
\hline $\begin{array}{l}\text { Índice de } \\
\text { Rugosidade do } \\
\text { Terreno (TRI) }\end{array}$ & $\begin{array}{l}\text { GDAL } \\
\text { (gdalogr:tri) }\end{array}$ & $\begin{array}{l}\text { nome do arquivo de saída (raster); computar as bordas da } \\
\text { imagem. }\end{array}$ \\
\hline Hidrografia & $\begin{array}{lr}\text { SAGA } & \text { (SAGA: } \\
\text { Sink Fill Sinks) e } \\
\text { SAGA (SAGA: } \\
\text { Channel Network } \\
\begin{array}{ll}\text { and Drainage } \\
\text { Basins) }\end{array} \\
\end{array}$ & $\begin{array}{l}\text { definir os nomes dos arquivos de saída para: bacias } \\
\text { hidrográficas (raster e vetor); Canais de fluxo (vetor); Junções } \\
\text { dos canais (vetor). }\end{array}$ \\
\hline
\end{tabular}

Fonte - Elaborado pelos autores.

\section{RESULTADOS}

Para atender as necessidades do plugin proposto neste trabalho, denominado plugin "MDE", as operações de processamento foram divididas em janelas (páginas diferentes) com a ajuda do software Qt Designer. A divisão das operações teve como princípio a segregação de conteúdos para os usuários. Ainda, almejou-se agrupar operações semelhantes. Contudo, os parâmetros de processamento foram dispostos na interface de modo que o usuário possua maior controle das operações realizáveis no plugin. Após realizar o projeto da interface no software Qt Designer e implementar o algoritmo de processamento no software Atom, o complemento desenvolvido foi aberto no QGIS para validar os resultados alcançados (Figuras 2 a 5). 
Fabrício Rosa Amorim Marcio Augusto Reolon Schmidt

Desenvolvimento de complemento QGIS para processamento de Silvana Phillipi Camboim

Niédja Sodré de Araújo mapas morfométricos

Luciene Stamato Delazari

Figura 2 - Interface inicial do plugin "MDE": parâmetros iniciais e parâmetros de saída para o processamento.

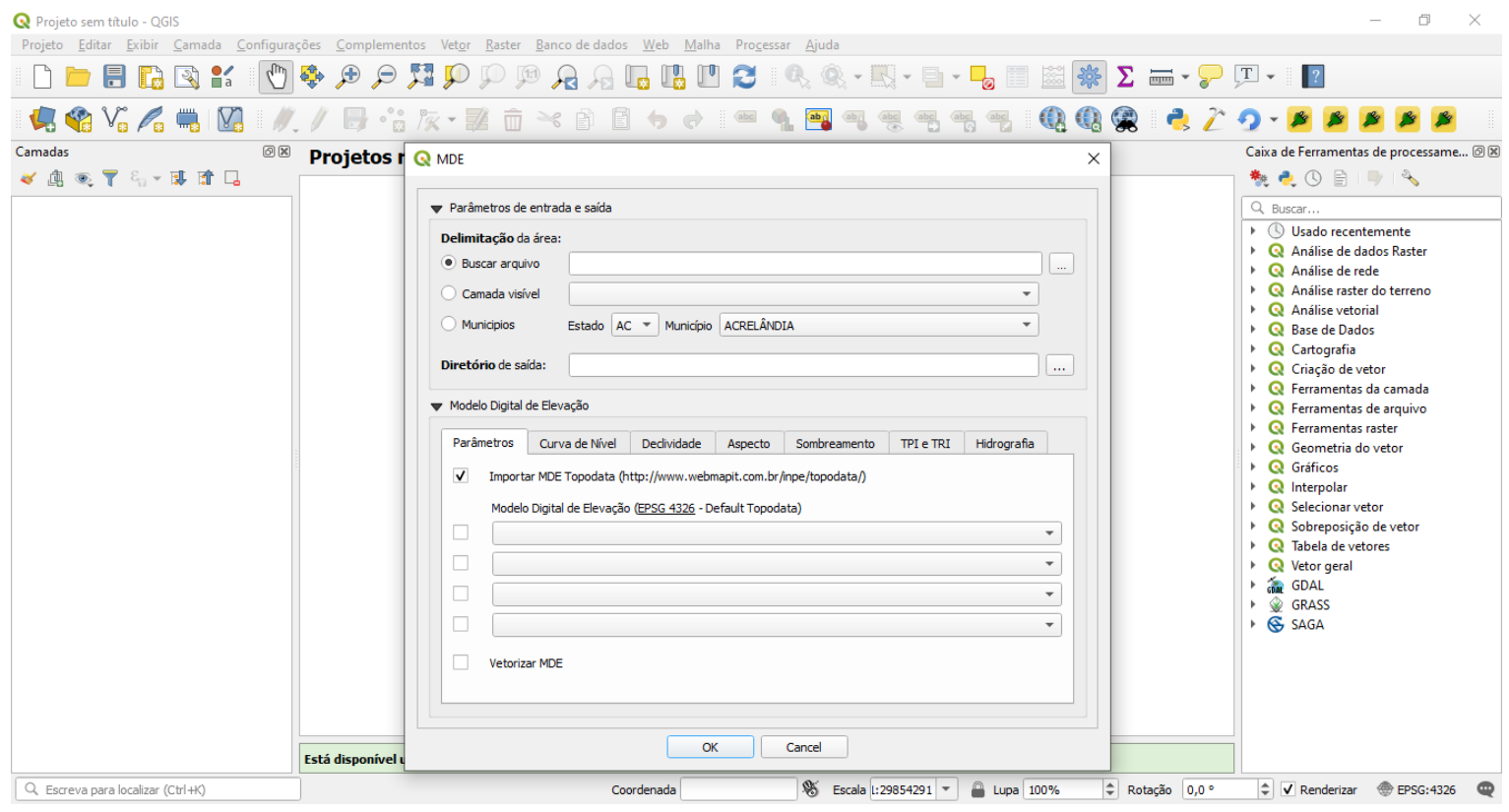

Fonte - Elaborado pelos autores.

Figura 3 - Interfaces do plugin "MDE": curvas de nível (A); declividade (B).

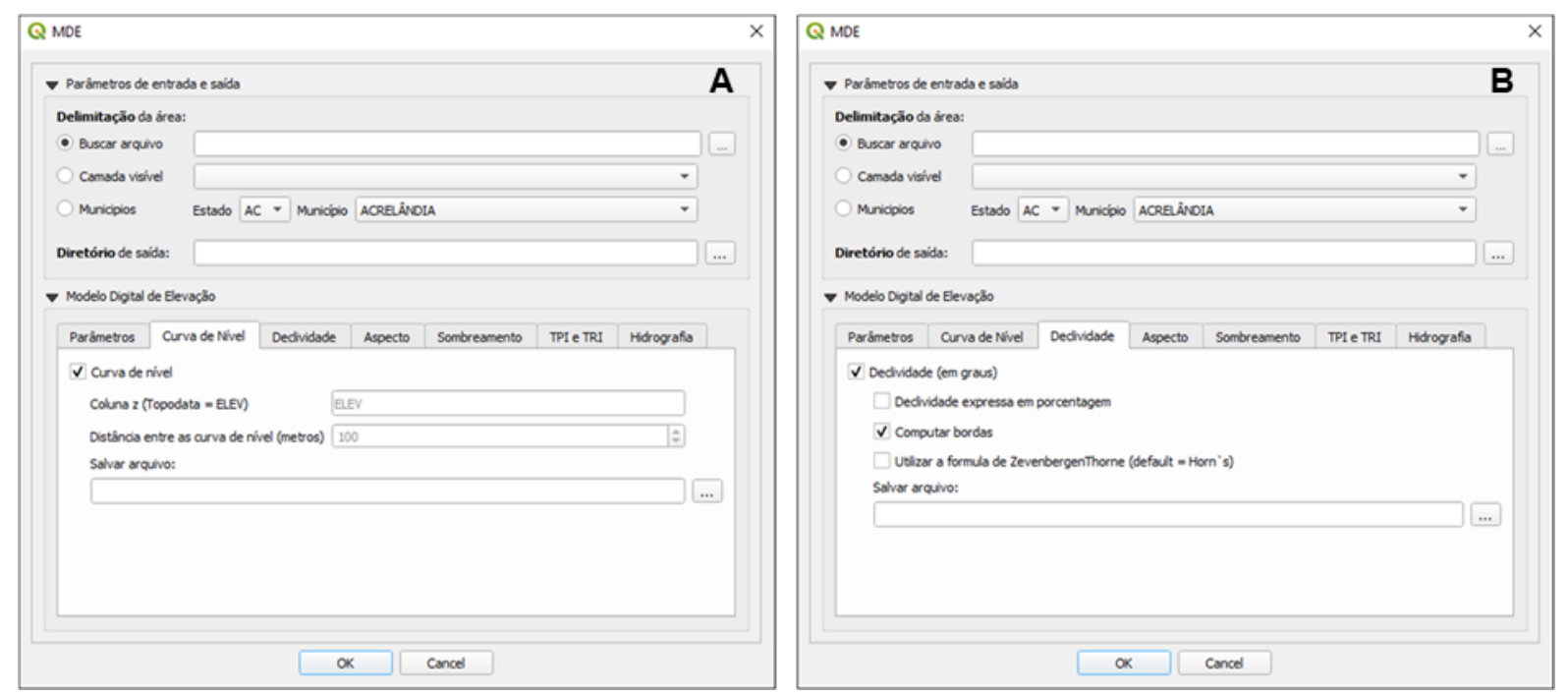


Fabrício Rosa Amorim Marcio Augusto Reolon Schmidt

Desenvolvimento de complemento QGIS para processamento de Silvana Phillipi Camboim mapas morfométricos

Niédja Sodré de Araújo Luciene Stamato Delazari

Figura 4 - Interfaces do plugin "MDE”: aspecto (A); sombreamento (B).

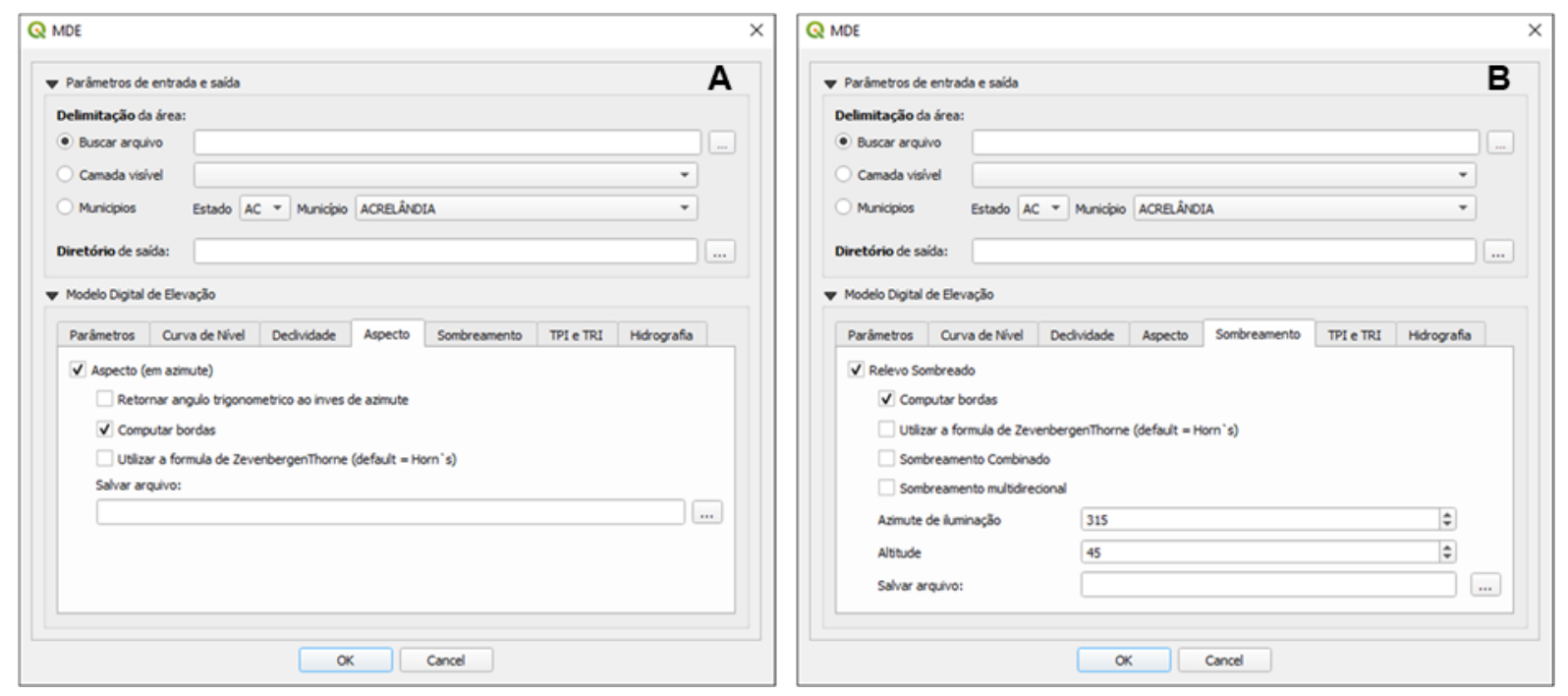

Figura 5 - Interfaces do plugin "MDE”: Índice de Posição Topográfica (TPI) e Índice de Rugosidade do Terreno (TRI) (a); Hidrografia (b).

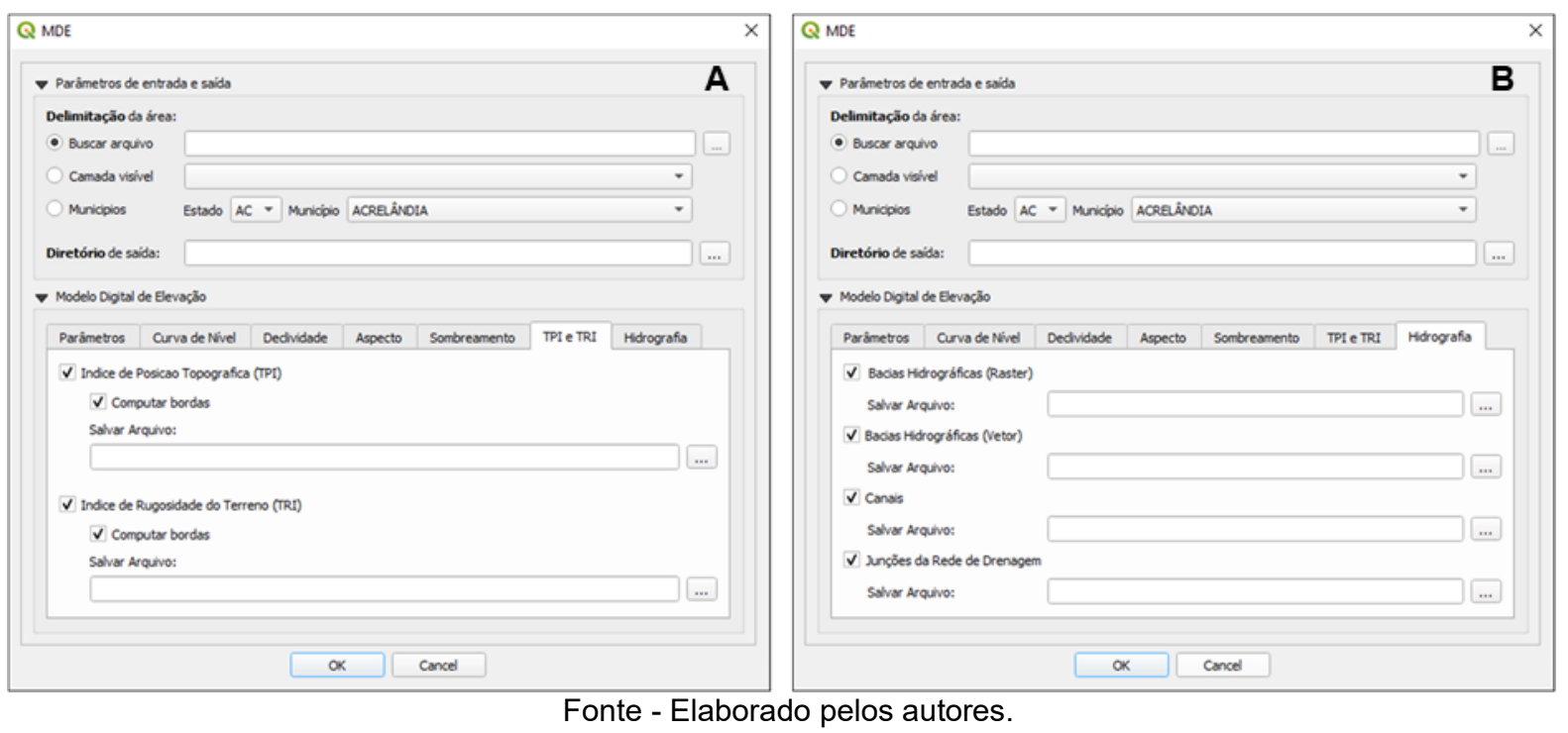


Fabrício Rosa Amorim Marcio Augusto Reolon Schmidt Silvana Phillipi Camboim

Desenvolvimento de complemento QGIS para processamento de Niédja Sodré de Araújo mapas morfométricos

\section{Validação dos resultados}

Após a finalização do desenvolvimento do plugin "MDE", selecionou-se o município de Uberlândia (MG) para validar a implementação do sistema. Como resultado, a Figura 6 e a Figura 7 apresentam os mapas projetados a partir da utilização do complemento desenvolvido neste trabalho. Os mapas são apresentados conforme a ordem em que aparecem na interface do complemento.

Figura 6 - Uberlândia (MG): Resultado do processamento realizado pelo Plugin "MDE" na tela do QGIS.

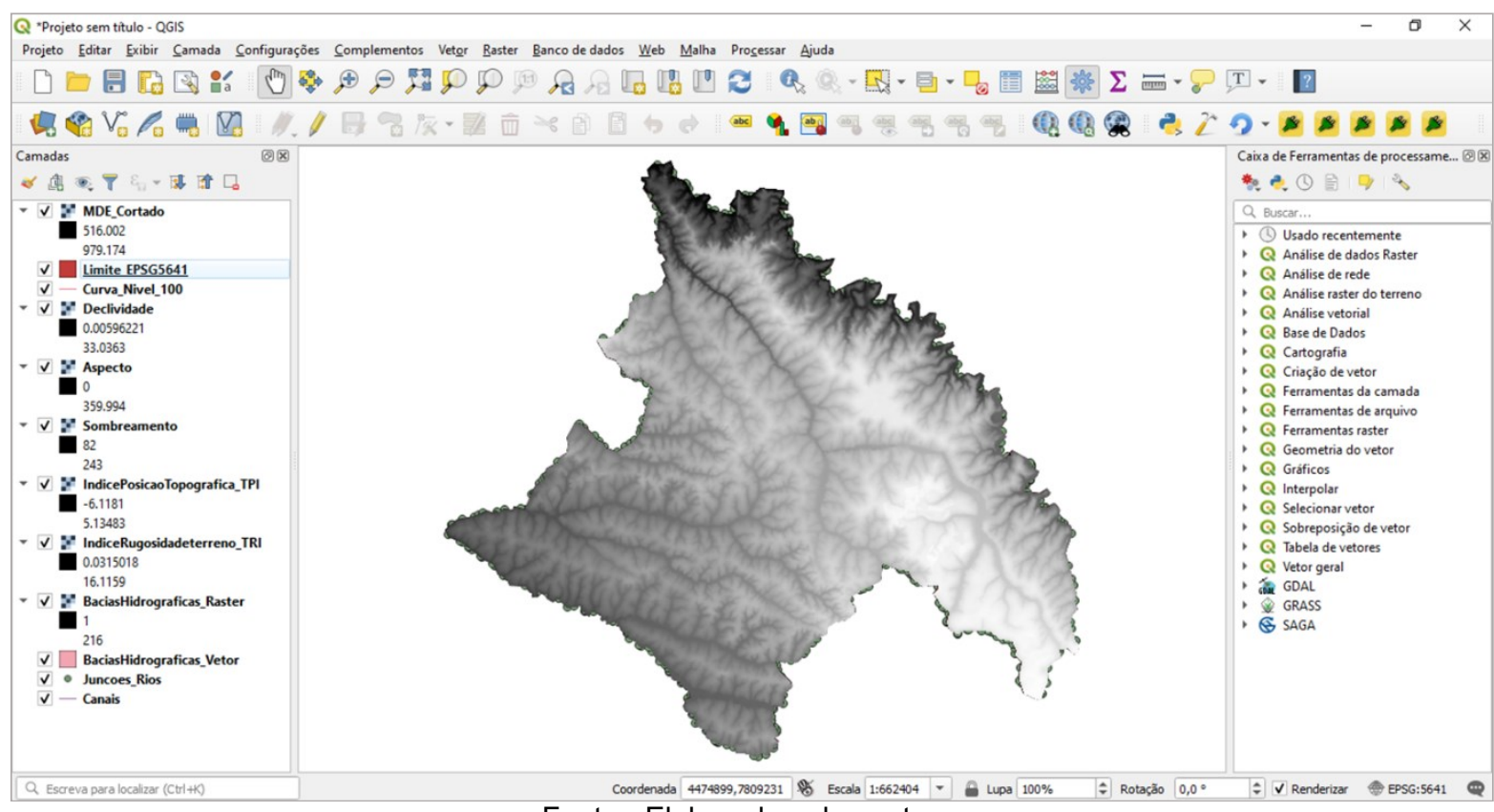

Fonte - Elaborado pelos autores. 
Fabrício Rosa Amorim

Marcio Augusto Reolon Schmidt Silvana Phillipi Camboim Niédja Sodré de Araújo

Desenvolvimento de complemento QGIS para processamento de mapas morfométricos

Figura 7 - Uberlândia (MG): Resultados individuais do processamento realizado pelo Plugin "MDE"

(Modelo Digital de Elevação Topodata (A); curvas de nível (B); declividade (C); aspecto (D);

sombreamento (E); Índice de Posição Topográfica -TPI (F); Índice de Rugosidade do Terreno - TRI

$(\mathrm{G})$; Bacias Hidrográficas formato raster $(\mathrm{H})$; Bacias Hidrográficas formato vetorial (I); Junções dos

Rios (J); Fluxos Hídricos (K)).

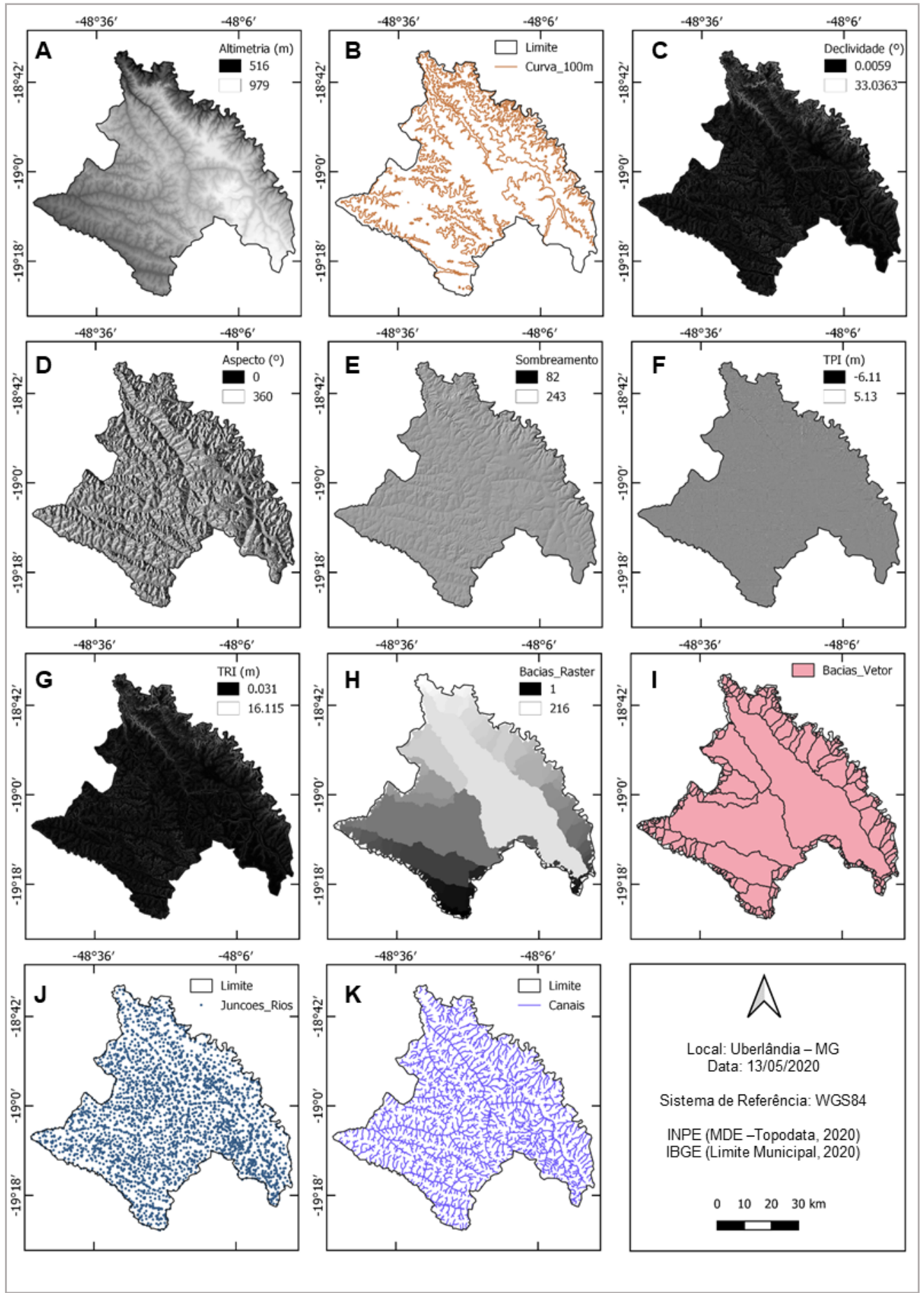

Fonte - Elaborado pelos autores. 


\section{CONSIDERAÇÕES FINAIS}

Este trabalho apresentou a proposta metodológica para desenvolvimento de uma ferramenta geoespacial para aquisição, tratamento e processamento de MDE em ambiente SIG. A partir dos dados topográficos do MDE Topodata, foi possível processar semiautomaticamente mapas morfométricos. Como resultado do projeto e produção de um complemento para o QGIS, obteve-se diferentes variáveis passíveis de extração automática em SIG. Ressalta-se, essas variáveis podem dar suporte à classificação multivariada da paisagem, fornecendo uma segmentação da mesma em ambientes topográficos. Ainda, a aquisição e processamento dos dados de altitude por meio de uma única ferramenta possibilita simplificar o processo de produção dos mapas de relevo. O plugin desenvolvido ainda carece de ajustes, futuras etapas podem complementá-lo, por exemplo: fornecer a simbologia dos dados de saída; fornecer outras opções de varáveis para o processamento; fornecer outras opções de resultados; e diminuir o tempo de processamento dos dados. $\mathrm{O}$ arquivo final do plugin desenvolvido nesse trabalho, denominado "MDE", está disponibilizado no sítio GitHub (https://github.com/CartoFabricio/MDE) para download como um arquivo compactado. Após o download, para instalá-lo no QGIS é necessário acessar a aba Complementos -> Gerenciar e Instalar Complementos -> Instalar a partir do ZIP -> Selecionar o arquivo na pasta fonte -> clicar em "Instalar o complemento". Após a instalação, o plugin "MDE" estará disponível na tela principal do QGIS para a sua utilização.

\section{Agradecimentos}

À Coordenação de Aperfeiçoamento de Pessoal de Nível Superior - CAPES pelo apoio financeiro para a realização da pesquisa, processo $\mathrm{n}^{\circ}$ 4001016002P6. Ao CNPq pela concessão de Bolsa Produtividade em Pesquisa, Processo 310312/2017-5 e pelo apoio financeiro para a realização da pesquisa, processos n $158303 / 2018-0$ e $n^{\circ} 142253 / 2020-0$.

\section{REFERÊNCIAS}

BRASIL. INPE - Instituto Nacional de Pesquisas Espaciais Topodata: banco de dados geomorfométricos do Brasil. INPE, São José dos Campos, Brasil, 2008. Disponível em: http://www.dsr.inpe.br/topodata. Acesso em: 03 de maio de 2020.

DENT, D.; YOUNG, A. Soil survey and land evaluation. London, Allen and Unwin. p. 278, 1981.

DE SMITH, M. J.; GOODCHILD, M. F.; LONGLEY, P. A. Geospatial analysis. Troubador publishing Itd, 2007.DRĂGUT, L., EISANK, C. Object representations at multiple scales from digital elevation

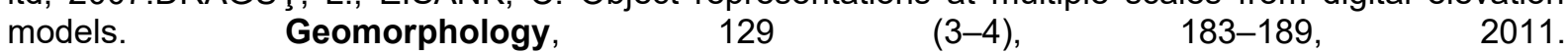
https://doi.org/10.1016/i.geomorph.2011.03.003

EL-SHEIMY, N.; VALEO, C.; HABIB, A. Digital terrain modeling: acquisition, manipulation, and applications. Artech House, 2005.

GILES, P.T.; FRANKLIN, S.E. An automated approach to the classification of the slope units using digital data. Geomorphology, v.21, p251-264. 1998. https://doi.org/10.1016/S0169-555X(97)00064-0

GOBERT, J. D. The effects of different learning tasks on model-building in plate tectonics: Diagramming versus explaining. Journal of Geoscience Education, v. 53, n. 4, p. 444-455, 2005. https://doi.org/10.5408/1089-9995-53.4.444

GUERRA, A.T.; GUERRA, A.J.T. Novo dicionário geológico-geomorfológico. 3. ed. Rio de Janeiro: Bertrand Brasil, 2003. 652p.

HENGL, T.; REUTER, H. Geomorphometry: concepts, software, aplications. Amsterdam: Elsevier, 765p, 2009.

IBGE - Instituto Brasileiro de Geografia e Estatítica. Organização do território. Disponível em: https://www.ibge.gov.br/geociencias/organizacao-do-territorio/estrutura-territorial/15774malhas.html?=\&t=downloads. Acesso em: 06 de maio de 2020.

IMHOF, E. Cartographic relief presentation. Walter de Gruyter GmbH \& Co KG, 2015.

LANG, S.; BLASCHKE, T. Análise da paisagem com SIG. Oficina de Textos, 2009. 
MCMASTER, K. J. Effects of digital elevation model resolution on derived stream network positions. Water Resources Research, v.38, n. 4, p.1-8, 2002. https://doi.org/10.1029/2000WR000150

MIZUKOSHI, H.; ANYIA, M. Use of contour-based DEMs for deriving and mapping topographic attributes. Photogrammetric Engineering and Remote Sensing, v.68, n.1, p.83-93, 2002.

MOORE, I. D., LEWIS, A., GALLANT, J. C. Terrain attributes: estimation methods and scale eff ects. In: Jakeman, A. J.; Beck, M. B.; McAleer, M. J. Modelling change in environmental systems. John Wiley e Sons Ltd. (Ed.), Chichester (Eng.). 584p. Capítulo 8, p.189-214, 1993.

QGIS.org. QGIS Geographic Information System. Versão 3.12. Open Source Geospatial Foundation Project, 2020. Disponível em: https://www.qgis.org/en/site/forusers/download.html. Acesso em: 06 de março de 2020.

ROBINSON, A. H.; MORRISON, J. L.; MUEHRCKE, P. C.; KIMERLING, A. J.; GUPTILL, S. C. Elements of Cartography. John Wiley \& Sons, 6 ed., 1995.

VALENZUELA, C. R. Data analysis and models. In: Belward, A. S. Valenzuela, C. R. Remote Sensing and Geographical Information Systems for Resource Management in Developing Countries. Euro Courses: Remote Sensing, Netherlands: Kluwer, v.1, cap.18, p.335-48, 1991.

VALERIANO, M. M. Modelos digitais de elevação de microbacias elaborados com krigagem. São José dos Campos, SP: INPE: Coordenação de Ensino, Documentação e Programas Especiais (INPE9364-RPQ/736). 54p., 2002a.

Programação do cálculo da declividade em SIG pelo método de vetores ortogonais. Espaço e Geografia (UnB), Brasília, DF, v. 5, n. 1, p. 69-85, 2002b.

Mapeamento da declividade em microbacias com sistemas de informação geográfica. Revista Brasileira de Engenharia Agrícola e Ambiental, Campina Grande, PB, v. 7, n. 2, p. 303-310, 2003. https://doi.org/10.1590/S1415-43662003000200020

Modelo digital de variáveis morfométricas com dados SRTM para o território nacional: o projeto Topodata. In: SIMPÓSIO BRASILEIRO DE SENSORIAMENTO REMOTO, 12, 2005, Goiânia. Anais... . Goiânia, 2005, p. 1-8.

Topodata: Guia de utilização de dados geomorfométricos locais. São José dos Campos: INPE, 2008. Disponível em: http://www.dsr.inpe.br/topodata/data/TDkrig.pdf. Acesso em: 02 de maio de 2020.

VALERIANO, M. M.; CARVALHO JÚNIOR, O. A. Geoprocessamento de modelos digitais de elevação para mapeamento da curvatura horizontal em microbacias. Revista Brasileira de Geomorfologia, Goiânia, GO, v. 4, n. 1, p. 17-29, 2003. https://doi.org/10.20502/rbg.v4i1.17

VALERIANO, M. M.; ROSSETTI, D. F. Topodata: Brazilian full coverage refinement of SRTM data. $\begin{array}{llllll}\text { Applied } \quad \text { Geography } & \text { (Sevenoaks), } & \text { v. } & 32, & \text { p. } & 300-309,\end{array}$ https://doi.org/10.1016/j.apgeog.2011.05.004

VALERIANO, M. M.; ROSSETTI, D. F.; ALBUQUERQUE, P. C. G. Topodata: desenvolvimento da primeira versão do banco de dados geomorfométricos locais em cobertura nacional. In: SIMPÓSIO BRASILEIRO DE SENSORIAMENTO REMOTO, 14, 2009, Natal. Anais... . Natal, 2009, p. 5499-5506.

WILSON, J. P.; GALLANT, J. C. Digital terrain analysis. Terrain analysis: principles and applications, v. 6 , n. 12, p. 1-27, 2000.

\footnotetext{
${ }^{\mathrm{i}}$ Recebido em: 24/06/2020

Aceito para publicação em: 20/08/2020
} 